\title{
Induced autokinesis
}

\section{LEONARD BROSGOLE ${ }^{1}$}

U. S. NAVAL TRAINING DEVICE CENTER

The egocentric location of a fixated visual stimulus was shifted away from the apparent median plane through induced movement. When the stimulus appeared to lie in the periphery of vision, the inducing frame was occluded resulting in auto kinesis toward the phenomenally straight-ahead position. This effect was termed "induced autokinesis." It was used in demonstrating that apparent egocentric displacement is sufficient for initiating autokinesis.

Question may be raised as to the nature of the relationship between perceived displacement and apparent motion. On the one hand, it may be contended that the two factors are causally related, where movement is the perceptual outcome of perceived displacement. On the other hand, it has been commonly held that although movement and displacement are usually associated, they are independent phenomena and the perception of one does not demand the other (e.g., Gregory \& Zangwill, 1963, p. 258). Induced motion and autokinesis are alleged instances of apparent motion with no egocentric displacement.

Regarding the phenomenon of induced movement, Brosgole (1966) has shown that a displacing background generated a dynamic Roelofs effect, i.e., the center of a luminous frame, presented in total darkness, was seen as straight-ahead even when it displaced back and forth over $24^{\circ}$ of visual arc. The frame appeared to remain more or less at rest, as its center seemed straight-ahead for the most part. An objectively stationary target, placed within the frame, appeared to move in a direction opposite to the true motion of the visual surround because it continuously changed location with respect to the apparent straight-ahead direction in space. Induced motion was therefore interpreted as the apparent displacement of a target relative to the egocentric or body system as a point of reference. It was suggested that perhaps this factor of subjective spatial displacement is also primary in the perception of autokinesis. Accordingly, the purpose of this study was to inquire whether or not an experimentally induced change in the apparent location of a visually isolated target is sufficient to generate autokinesis in a prescribed direction.

\section{EXPERIMENT I}

In the typical induced motion paradigm, $\mathrm{S}$ fixates a target located in the objective median plane while a surrounding frame displaces back and forth. As the center of the frame travels away from the objective straight-ahead position, the target not only seems to move to the side, but it also appears to change its egocentric location. That is to say, it is clearly seen to drift off to the left side of the testing room when the frame displaces to the right and to glide to the right side of the room as the frame journeys to the left.

When the procedure is somewhat varied by requiring $S$ to adjust the target so as to keep it continuously straight-ahead, the apparent median plane is found to displace along with the frame.

It logically follows that since Ss are fairly accurate in localizing the straight-ahead in total darkness, the effect that an asymmetrically located visual frame has upon the apparent straight-ahead should gradually dissipate once that frame has been occluded. In other words, the Roelofs effect generated by a frame displacing to the right, for example, should slowly diminish with the straight-ahead returning to a true center position, if that frame were extinguished just prior to reversing and going to the left.

A fixated stationary target, then, which has been induced by the frame to assume an apparent position to S's left, should now be seen to autokinetically float back toward center as it draws progressively closer and closer to the phenomenally straight-ahead position.

The first experiment is directed, therefore, at determining the effect of extinguishing an asymmetrically extending field upon the apparent median plane, as well as, to establish whether or not autokinesis can be induced in the manner specified.

\section{Method}

Apparatus. The apparatus consisted of a wooden frame, 5-1/2 ft. high and $6 \mathrm{ft}$. long, a variable speed DC motor, a system of pulleys and a horizontally traveling screen (60 in. high $\times 30$ in. wide) upon which stimuli could be mounted. The screen was driven back and forth along a set of tracks over a distance of 22 in. by the motor via the pulley system. Once reaching the end of its 22-in. journey, the screen activated a relay reversing the motor and, as a result, its direction of travel. Thus, once the remotely operated motor was started, the screen took a continuous trip back and forth across space.

A Selsyns synchronous motor was mounted on the Ieft side of the apparatus with a pulley secured to its shaft. A 10 turn, linear potentiometer, with a pulley of the same specification anchored to its shaft, was fastened to the right side of the apparatus. A visual target was hooked onto a belt which joined the two pulleys.

S remotely controlled the Selsyns motor by turning a knob affixed to the shaft of a duplicate motor which 
was mounted in a minibox. Thus, within 10 turns of the control knob, $\mathrm{S}$ was able to laterally move the target 60 in. across space while simultaneously adjusting the potentiometer from $0-1000 \mathrm{ohms}$ resistance. A DC voltage was placed across the coil of the pot with leads from the armature and one terminal feeding through a Brush high gain DC amplifier into a Brush analog strip chart recorder. Changes in the horizontal location of the target effected by $S$ were, thereby, translated into voltage outputs and concurrently charted.

Stimuli. The stimuli consisted of a $3 / 8$ in. diameter circular spot of light surrounded by a $24 \times 30$ in. horizontal rectangular frame with $1-1 / 8$ in. wide borders. The circular target was fastened to the belt and, therefore, under S's control while the frame was attached to the screen varied by $E$. They were both constructed of Sylvania, white, electroluminescent Tape-Lite which basically served two advantages. For one, their brightness was regulated by varying the $\mathrm{AC}$ input with the target set at 1.8 and the frame at $.009 \mathrm{ft}$.L. And, for another, it permitted the design of circuitry which enabled the reversing mechanism in the apparatus to turn the frame and target on and off independently in a manner designated by $\mathrm{E}$ in advance of each trial.

Procedure. The experiment took place in total darkness with $\mathrm{S}$ seated $10 \mathrm{ft}$. from the apparatus. $S$ initially observed only the target for $2 \mathrm{~min}$. (Preautokinetic condition). S was told that he would be required to fixate the target which $\mathrm{E}$ could either move to the right, left or hold perfectly stationary. He was handed the control knob and informed that he, too, could control the motion of the spot of light. By turning the knob $\mathrm{CW}$, the spot would move to the right; by rotating it $\mathrm{CCW}$, the spot would go to the left. $\mathrm{He}$ was advised to ignore any up-down motion that $\mathrm{E}$ might arbitrarily impose upon the target and was instructed to attempt to keep the target directly straight ahead (i.e., perpendicular to the midline of his body) by turning the knob appropriately. So, for example, whenever movement to the left was detected, S's task was to return the spot to the right. After receiving his instructions, $S$ positioned the target to the apparent straight-ahead and the 2-min. inspection period commenced. The amount of lateral autokinesis observed in the spot was inferred from the compensatory adjustments recorded on the Brush.

In the second condition, the frame was turned on and continuously visible. With the frame stationary and its center in the objective median plane, $S$ adjusted the target to the apparent straight-ahead position. He was told that E could now move the background, as well as the target. S's task was to ignore the frame and continue to keep the spot straight ahead, just as before. The frame traveled back and forth through $10^{\circ} 20^{\prime}$ of visual arc at a rate of $20^{\prime} / \mathrm{sec}$. for one complete cycle; i.e., from center to right, back through center to left and then to center again. It took 31 sec. for it to go from extreme left to extreme right and vice versa. The initial direction in which the frame moved (right or left) was counterbalanced among Ss. S's adjustments provided an index of the amount of motion induced in the spot by its surround.

The third condition was identical to the second except that the initial movement of the frame was always to the right and it was extinguished upon reversing to the left. A measure of the amount of leftward motion induced in the spot, as well as the extent to which the target tended to autokinetically drift back to center over a $31 \mathrm{sec}$. interval was, thereby, obtained.

The fourth condition was the same as the third, except that the frame started to the left and went out when reversing to the right.

A post autokinetic session, identical to Condition 1 , terminated the experiment. Conditions 1 and 5 remained in fixed order with 2,3 and 4 randomized differently for each $\mathrm{S}$. After completion of the procedure, $\mathrm{S}$ was again run on Conditions 2 and 3 , but with no adjustments required. $S$ merely provided a running account of the apparent location and movement of both the target and frame.

Subjects. One male and seven females participated in this study. Ranging in age from 18-59, their mean age was 30.5 years. All were naive as to the purpose of the experiment.

\section{Results}

We sought to determine how far back toward center the target tended to drift autokinetically after being induced to move to the side and to ascertain whether or not this drift exceeded the extent of spontaneously initiated autokinesis during a comparable time interval. The scores subjected to statistical analysis were in terms of the total range of movement seen in each condition.

The disparity between the adjusted position of the target when the frame was at its extreme right, and when at its extreme left, represented the total amount of induced motion seen in Condition 2. The amount of induced motion seen to the left in Condition 3, where the frame displaced to the right, was added to that of Condition 4 in which the frame moved to the left resulting in an apparent motion of the spot to the right. This provided a means of determining if there was a fall off in induced motion due to the induced autokinetic procedure. The range of induced autokinesis was derived in the same fashion.

Since there were $31 \mathrm{sec}$. of induced autokinesis in each of Conditions 3 and 4, the first 31 sec. of inspection in the pre- and post autokinetic sessions were totaled so as to afford comparison data of similar derivation. But as the direction of phenomenal movement was invariably the same in the pre- and post conditions, the scores were summed ignoring sign. That is to say, it seemed inadvisable to calculate a range measure under such circumstances, for two quite 
sizeable movements may, at most, yield a negligible range if they are of almost equal magnitude in the same direction.

With regard to the results, there were $3^{\circ} 19^{\prime}$ of spontaneously initiated autokinesis, as against $7^{\circ} 13^{\prime}$ of induced motion in Condition 2, $6^{\circ} 47^{\prime}$ of induced motion summed across Conditions 3 and 4 , and $6^{\circ} 17^{\prime}$ of induced autokinesis. A significant difference was found when the treatments were simultaneously compared with a Friedman two way analysis of variance $\left(\mathrm{xr}^{2}=\right.$ 17.21, $\mathrm{p}<.001$ ). According to the Wilcoxon Signed Ranks Test, there was significantly less spontaneousiy initiated autokinesis than there was induced autokinesis $(\mathrm{T}=3.5, \mathrm{p}<.025)$, as well as, induced motion measured in the two ways previously specified (in each instance $T=0, p<.01)$. The other conditions did not differ reliably from one another. It is obvious, then, that induced autokinesis is essentially equal in magnitude, although opposite in direction, to induced motion and far in excess of spontaneously initiated autokinesis over the same period of time.

In addition, whereas spontaneously initiated autokinesis was found to be unidirectional, induced autokinesis was bidirectional. In other words, the induced autokinetic procedure was able to overcome S's propensity to see movement in one preferred direction, as indicated in both the pre- and post conditions, so as to yield a movement in the opposite or nonpreferred direction. Having established the legitimacy of the induced autokinetic phenomenon, let us examine the phenomenological protocols obtained after the main procedure with the target left straight ahead and no adjustment required.

Every S reported, whenever the frame went out, that the target appeared to be located off to the side. Seven of the eight Ss saw the spot float back toward center very very slowly. Four of them saw a straight lateral movement with the target sometimes overshooting center and bouncing back. At times the spot would drift upward once reaching a central position. Two Ss saw linear movement along a $45^{\circ}$ meridian-up and toward the straight-ahead-and one saw a parabolic type of motion, i.e., predominantly upward initially followed by an arching over toward center and finally descending when nearing the apparent median plane.

Contrary to these reports, the eighth $S$ tended to see a slight movement away from center after extinction of the frame. This deflated the induced effect reported earlier. Since this $S$ attributed all $10^{\circ} 20^{\prime}$ of movement to the target in the induced motion conditions, it was reasoned that perhaps the frame did not shift her straight-ahead laterally to its fullest possible extent. If so, there would be no requirement for the apparent median plane to return to its veridical location. In order to test this premise, $\mathrm{S}$ was seated at $1 / 2$ the distance to the apparatus on the following day. The frame now displaced $10^{\circ} 20^{\prime}$ to either side of the objective median plane, or a total of $20^{\circ} 40^{\prime}$. As a result, only about $16^{\circ}$ of displacement was attributed to the target and autokinesis was induced in the expected direction. Our hypothesis was, therefore, substantiated.

\section{EXPERIMENT II}

The function of this study was to question whether induced target movement is necessary for generating induced autokinesis, or if merely changing the apparent location of the target is sufficient. In other words, does the spot have to be seen to move off to the side in order to return to center?

\section{Method}

Procedure. The displacing frame was used to produce a Roelofs effect, i.e., to laterally shift the apparent median plane, with no target present. With the frame centered, $S$ was instructed to fixate and maintain a straight-ahead gaze ignoring the presence of the frame. The field then moved to the right in one instance and to the left in another with the two conditions counterbalanced among Ss. Upon reversing the frame was extinguished. The spot appeared in the objective median plane $1 / 2$ sec. later. S's task was to furnish a continuous verbal accounting of where the spot appeared and how it seemed to move.

Subjects. Three males and five females took part in this study. Ranging in age from 18-51, their mean age was 24.1 years. They were all undergraduate students from $\mathrm{C}$. W. Post College who were naive as to the purpose of this experiment.

\section{Resulis}

The phenomenological findings of the previous study were unanimously supported. When the frame was extinguished, the target invariably seemed to appear in the periphery and creep toward a central position. We even came across one $S$ (at long last) who superimposed a downward movement upon the apparent lateral course of the spot.

In addition to demonstrating that induced movement need not necessarily precede induced autokinesis, these findings definitely indicate that induced motion is an egocentrically determined phenomenon which is not contingent upon the displacement of a visual target relative to its surround.

\section{EXPERIMENT III}

Carr (1910) and Gregory and Zangwill (1963, pp. 253-255) suggest that whenever an isolated visual stimulus either objectively or phenomenally off center is fixated, an eye strain ensues. Returning the eye to center yields an autokinesis away from the direction of strain or toward the median plane. It could be argued that the induced autokinetic procedure fulfills these requirements. Induced autokinesis may, therefore, be an artifact of induced eye strain and not at 
all related to changes in the egocentric location of the target. Accordingly, the aim of this study was to explore such a proposal.

\section{Method}

Procedure. The lateral extent of motion induced in the target by the frame was ascertained for each $\mathrm{S}$ by means of the procedure employed in Condition 2 of the first experiment. Then, over three counterbalanced trials, the spot was placed either off to the left, right or straight ahead. The three placements corresponded to the adjusted positions of the target when the frame was at its extremes and centered in the above mentioned induced motion condition. The spot was presented in one of the three locations surrounded by the frame, which was centered, so that when the spot was straight ahead, it looked straight ahead; when it was offset, it looked displaced to the side.

The frame was then extinguished and S's task was to hold it in that position-to cancel out any lateral autokinesis-through the adjustment procedure for 31 sec. The three trials were followed by three noadjustment trials.

In summary, the purpose of this paradigm was to determine the direction of apparent target movement from those positions in which it would have appeared at the onset of induced autokinesis, as well as straightahead, but with no deception involved regarding its true location.

Subjects. Five female undergraduate students from C. W. Post College served as Ss. Their mean age was 20.0 years with a range of from 18-22.

\section{Results}

All five Ss saw movement in one preferred direction (three to the right and two to the left) regardless of target location. Such unanimity is significant at the $5 \%$ level according to the Binomial test. Since stimulus offset had no differential effect upon autokinesis, we categorically reject the eye strain hypothesis in favor of one based upon change in egocentric target location.

\section{DISCUSSION}

The foregoing results clearly demonstrate that apparent location change is sufficient for generating autokinesis. They are consistent with the findings of Mandel (1963) who related Sandstrom's 'luminous point phenomenon" to problems of perceptual localization; Cormack (1963) who found a high correlation between the degree of autokinesis and the extent to which the apparent starting location of the stimulus displaced; and Glick, Wapner, and Werner (1965), who indicated that upward autokinesis is accompanied by downward shifts in the apparent eye level position.

Although displacement and movement usually go together, the question arises whether or not location change is necessary in order to initiate autokinesis.
Moustgaard (1963) has powerfully defended a negative point of view, citing instances of paradoxical autokinesis, i.e., where the autokinetic experience was not accompanied by an apparent displacement of the fixated stimulus. We, too, have observed this phenomenon in the laboratory but are not yet prepared to call it paradoxical, for there seem to be several different classes of events which underlie this effect.

For one, evidence of such a paradox often emerges when data regarding movement and displacement are gathered independently or successively; rarely does this occur when a response to both factors is simultaneously required. It is as if some Ss regard movement and displacement as two distinctively different characteristics of the same stimulus or, rather, that they tend to be field oriented when attempting to detect motion and body oriented when estimating location change. The paradox may, therefore, represent different strategies adopted by $S$ in trying to complete the requirements of the experiment.

Secondly, $\mathrm{S}$ sometimes gains the impression that his body is moving in the same direction as the autokinetic stimulus, in which case there is the sensation of spatial displacement with, of course, no egocentric displacement. In these instances the appearance of a paradox may reflect $E$ 's failure to recognize that $S$ is responding from within an egocentric rather than an external frame of reference.

Thirdly, Ss usually feel that they are tracking the autokinetic stimulus with their eyes. Some occasionally report that although the target appears to move, it continues to remain straight ahead. Further questioning usually reveals that when the eyes or head are no longer phenomenally aligned with the trunk, $S$ oscillates between body parts in determining perceived direction. As a result, the target may be referred to as straight ahead because it stimulates the fovea, even though the eye is phenomenally turned.

Finally, we have personally experienced what more closely approximates pure paradoxical autokinesis. The fixated target appears to be energized, possessing intensity and direction. It strains at the leash, so to speak, but somehow cannot break loose. A simple isometric analogy is forcing one's palm against the wall and maintaining the pressure. Although the dynamics of the situation readily reveal themselves, the arm appears static until the restraining barrier is removed. In the same fashion, we are not quite ready to conclude that the autokinetic stimulus appears to move. It certainly does not seem to be at rest, but this does not necessarily imply movement. Perhaps the problem partially resides within our language which dichotomizes rest and motion. The absence of rest, however, is action or activity, not motion.

Since paradoxical movement has not yet been decisively demonstrated, we may conclude that egocentrically perceived location change is at least sufficient and possibly necessary for initiating autokinesis. 


\section{References}

Brosgole, L. An analysis of induced motion. U. S. Naval Training Device Center, Tech. Rep., IH-48, February, 1966.

Carr, H. A. The autokinetic sensation. Psychol. Rev., 1910, 17, 42-75.

Cormack, R. H. Ocular tracking as a measure of autokinetic move ment. Percept. mot. Skills, 1963, 17, 223-226.

Glick, J. A., Wapner, S., \& Wemer, H. Some relations between autokinetic motion and space localization. Acta Psychol., 1965, $24,41-48$

Gregory, R. L., Z Zangwill, O. L. The origin of the autokinetic effect. Quart. J. exp. Psychol., 1963, 15, 252-261.
Mandel, I. J. Some determinants of the manual location of an isolated visual stimulus. Paper read at Eastern Psychological Association, New York, 1963.

Moustgaard, K. A phenomenological approach to autokinesis Scand. J. Psychol., 1963, 4, 17-22.

\section{Note}

1. The author expresses his gratitude to $R$. Cristal and $\mathrm{S}$. Jordan for their most helpful suggestions and assistance.

(Accepted for publication November 25, 1966.) 